\title{
An Innovative Cell Microincubator for Drug Discovery Based on 3D Silicon Structures
}

\author{
Francesca Aredia, ${ }^{1,2}$ Francesca Carpignano, ${ }^{3}$ Salvatore Surdo, ${ }^{4}$ Giuseppe Barillaro, ${ }^{4}$ \\ Giuliano Mazzini, ${ }^{1,2}$ Anna Ivana Scovassi, ${ }^{1}$ and Sabina Merlo ${ }^{3}$ \\ ${ }^{1}$ Istituto di Genetica Molecolare (IGM-CNR), 27100 Pavia, Italy \\ ${ }^{2}$ Dipartimento di Biologia e Biotecnologie "L. Spallanzani", Università di Pavia, 27100 Pavia, Italy \\ ${ }^{3}$ Dipartimento di Ingegneria Industriale e dell'Informazione, Università di Pavia, 27100 Pavia, Italy \\ ${ }^{4}$ Dipartimento di Ingegneria dell'Informazione, Università di Pisa, 56122 Pisa, Italy \\ Correspondence should be addressed to Sabina Merlo; sabina.merlo@unipv.it
}

Received 9 October 2015; Revised 3 January 2016; Accepted 13 January 2016

Academic Editor: Yu-Lun Chueh

Copyright (C) 2016 Francesca Aredia et al. This is an open access article distributed under the Creative Commons Attribution License, which permits unrestricted use, distribution, and reproduction in any medium, provided the original work is properly cited.

\begin{abstract}
We recently employed three-dimensional (3D) silicon microstructures (SMSs) consisting in arrays of $3 \mu \mathrm{m}$-thick silicon walls separated by $50 \mu \mathrm{m}$-deep, $5 \mu \mathrm{m}$-wide gaps, as microincubators for monitoring the biomechanical properties of tumor cells. They were here applied to investigate the in vitro behavior of HT1080 human fibrosarcoma cells driven to apoptosis by the chemotherapeutic drug Bleomycin. Our results, obtained by fluorescence microscopy, demonstrated that HT1080 cells exhibited a great ability to colonize the narrow gaps. Remarkably, HT1080 cells grown on 3D-SMS, when treated with the DNA damaging agent Bleomycin under conditions leading to apoptosis, tended to shrink, reducing their volume and mimicking the normal behavior of apoptotic cells, and were prone to leave the gaps. Finally, we performed label-free detection of cells adherent to the vertical silicon wall, inside the gap of 3D-SMS, by exploiting optical low coherence reflectometry using infrared, low power radiation. This kind of approach may become a new tool for increasing automation in the drug discovery area. Our results open new perspectives in view of future applications of the 3D-SMS as the core element of a lab-on-a-chip suitable for screening the effect of new molecules potentially able to kill tumor cells.
\end{abstract}

\section{Introduction}

Most of the knowledge in cancer biology is due to discoveries obtained by setting experimental conditions on twodimensional (2D) models, which fail to be extrapolated to in vivo conditions due to the three-dimensional (3D) complexity of tissues and organs. To bypass these inconveniences, there is an increasing interest in investigating cell cultures on 3D matrices, which were described as promising tools to monitor cell growth, proliferation, and transformation, thanks to their capability to better mimic the in vivo situation [1-4].

In this perspective, we developed three-dimensional silicon microstructures (3D-SMSs) formed by periodic arrays of parallel $\approx 3 \mu \mathrm{m}$-thick silicon walls separated by $\approx 5 \mu \mathrm{m}$-wide,
$50 \mu \mathrm{m}$-deep air gaps $[5,6]$. We demonstrated that these microdevices are suitable for the culture of human cancer cells $[7,8]$ in $3 \mathrm{D}$ with a proliferation rate similar to the 2D counterpart. Moreover, we provided the evidence that the devices are useful tools for analyzing the mechanical properties/plasticity of different human cancer cell lines, monitoring the spreading of the cells on the wall inside the gaps by means of label-free optical detection method [9]. Focusing on the possible use of microdevices in cancer cell biology, given that cancer still represents a major health problem worldwide requiring the development of more effective strategies, we investigated the behavior of several human tumor cell lines characterized by different biological aggressiveness. We recently provided the original evidence that the behavior of tumor cells on the 3D-SMS depends 


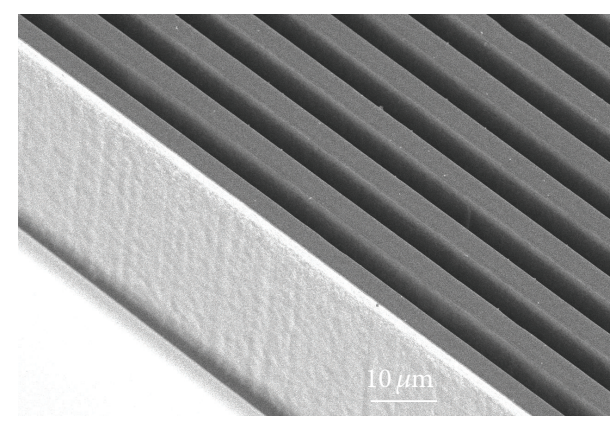

(a)

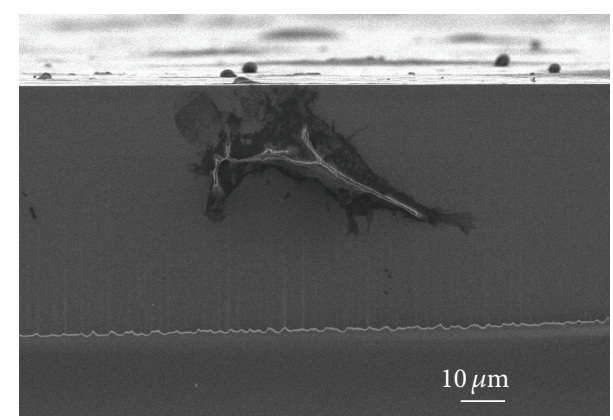

(b)

FIGURE 1: Scanning electron microscopy (SEM). (a) SEM bird view of electrochemically micromachined 3D-SMSs highlighting the high quality of the microfabricated structures in terms of both uniformity and low surface roughness. (b) SEM cross section view of a single cell cultured directly into a 3D-SMS; the cell capability of growing adherent to the vertical surfaces of the 3D-SMS is clearly evident.

on their aggressiveness, based on the quantification of the fraction of cells inside the gaps, over the total cell number [10].

Starting from the assumption that resistance to chemotherapy, a major obstacle for cancer eradication, is mainly related to an intrinsic failure in the activation of the apoptotic pathways of death [11-13], in the present study we have explored 3D-SMS for mimicking the in vivo situation and possibly finding a new tool for drug discovery. The 3D microenvironment will allow, in principle, to obtain results better related to the in vivo condition; of note, in this $3 \mathrm{D}$ microenvironment cell proliferation was very similar to that of the conventional 2D condition. Our experimental strategy aimed to exploit a peculiar property of apoptotic cells, related to the changes in cell morphology and adhesion properties [14-17]. In fact, cancer cells growing adherent to a substrate respond to an apoptogenic stimulus by shrinking their volume and modifying their adhesion molecules, thus floating into the medium. In this respect, we previously observed that under stress conditions, for example, extended culture times, cells grown on our 3D-SMS tended to detach from the walls and remain with a round shape on top of the walls [7]. We performed viability experiments aimed at verifying that cells entering the narrow deep gaps were alive at least after $72 \mathrm{~h}$ incubation time. This means that these cells were able to explore the vertical silicon walls and grow inside the narrow gaps with sufficient exchange of nutrients and oxygen. Cells were treated with the chemotherapeutic drug Bleomycin according to a previously reported protocol to induce apoptosis in other cancer cell lines [1820]. In this work, a preliminary evaluation of the apoptosis outcomes on 3D-SMS has been performed by means of conventional fluorescence microscopy analysis and several acquired fluorescence images are here reported as a result of our experiments. In view of future applications of 3D-SMSs as core elements of a lab-on-a-chip suitable for screening the effect of new molecules potentially able to kill tumor cells, we have investigated the possibility to perform label-free cell detection, by exploiting optical reflectometry. In previous works, we demonstrated the functionality of a compact fiber optic (FO) Michelson interferometer for time-domain
Optical Low Coherence Reflectometry (OLCR) that exploited infrared (IR) broadband radiation provided by a tungsten lamp for characterizing 3D-SMSs by measuring the optical path-length among several silicon/air interfaces for arrays with different spatial period, that is, 8,10 , and $20 \mu \mathrm{m}$ [2123]. In this paper, label-free detection of a cell adherent to the vertical silicon wall, inside the gap, of an $8 \mu \mathrm{m}$ periodic array is demonstrated with IR OLCR, a minimally invasive technique that could be implemented also on a chip for monitoring cell detachment due to apoptosis.

\section{Materials and Methods}

2.1. Fabrication of Silicon Microstructures. Three-dimensional silicon microstructures (3D-SMSs) of this work were fabricated by means of electrochemical micromachining technology (ECM), which is detailed in $[5,6,24]$. 3D-SMSs, consisting of vertical $\approx 3 \mu \mathrm{m}$-thick silicon walls periodically separated by $50 \mu \mathrm{m}$-deep and $\approx 5 \mu \mathrm{m}$-wide air gaps, were integrated within a circular region (surface area of $0.64 \mathrm{~cm}^{2}$ ) of a silicon die with size $1.5 \mathrm{~cm} \times 1.5 \mathrm{~cm}$. Electrochemically micromachined silicon dice were cut into two rectangular parts and reduced in size to fit in the wells of 12-well plates exploited during the subsequent cell culturing experiments. A peculiar property of these die consists in the coexistence of planar (2D) as well as three-dimensional supports: on the sides, 3D-SMS has flat silicon regions, similar to glass surfaces used in standard cell culture. This feature provides an intrinsic control for comparing the cell behavior in $2 \mathrm{D}$ and $3 \mathrm{D}$, side by side.

2.2. Scanning Electron Microscopy (SEM). The morphological characterization of the silicon microstructures, after the ECM process (i.e., empty 3D-SMS) as well as after cell culturing (i.e., 3D-SMS populated by cells), was performed by scanning electron microscope (JSM-6390 from JEOL, Inc.) at an acceleration voltage of $3 \mathrm{kV}$. Figure 1(a) shows a SEM bird view of electrochemically micromachined 3D-SMS highlighting the high quality of the microfabricated structures in terms of both uniformity and low surface roughness. Figure 1(b) presents 


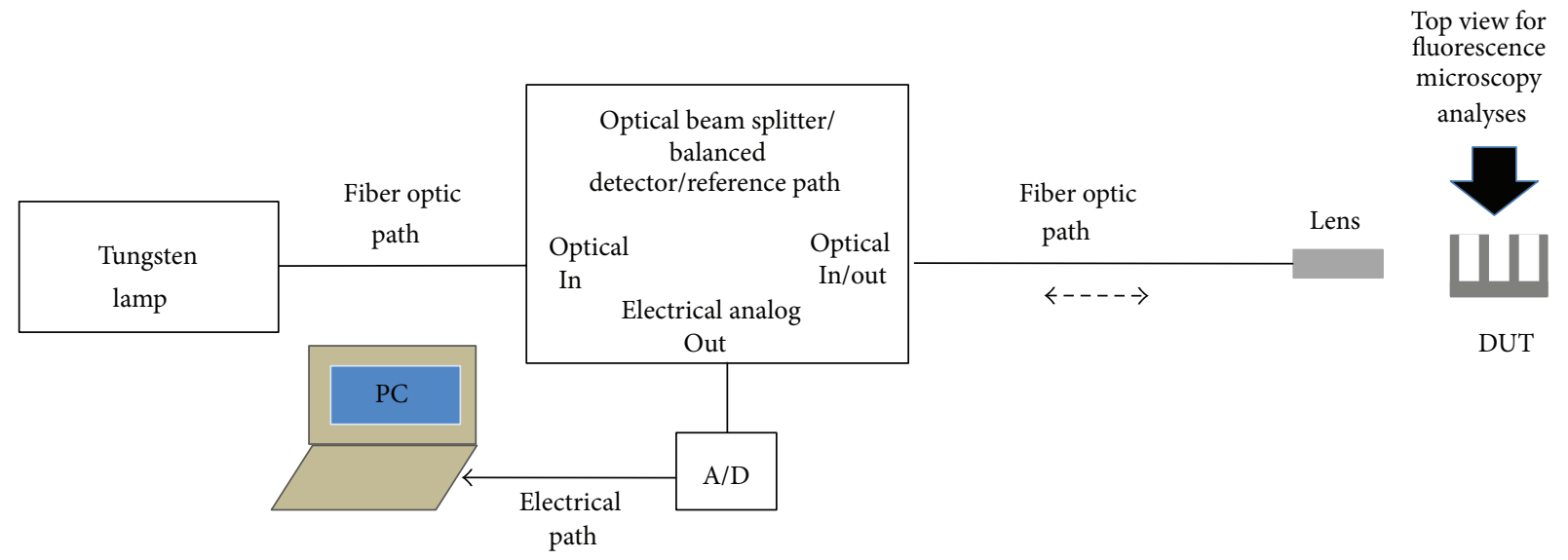

FIGURE 2: Instrumental configuration for the infrared low coherence reflectometer. DUT: device under test. A/D: analog to digital conversion board. PC: personal computer.

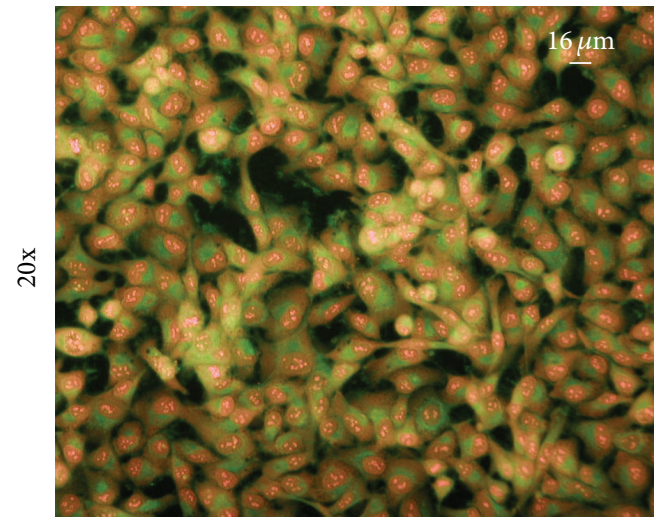

(a)



(c)

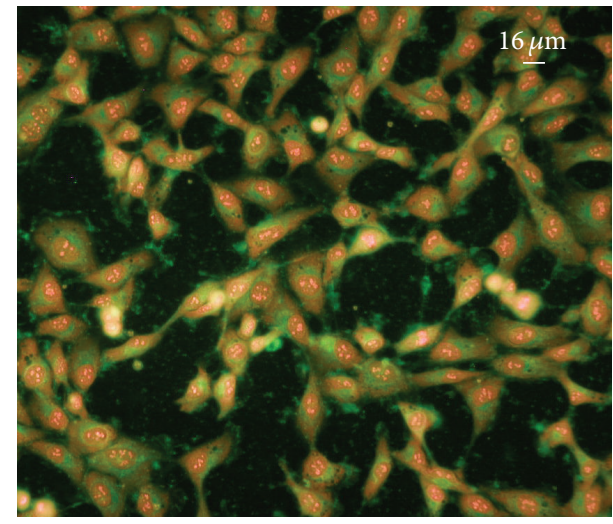

(b)

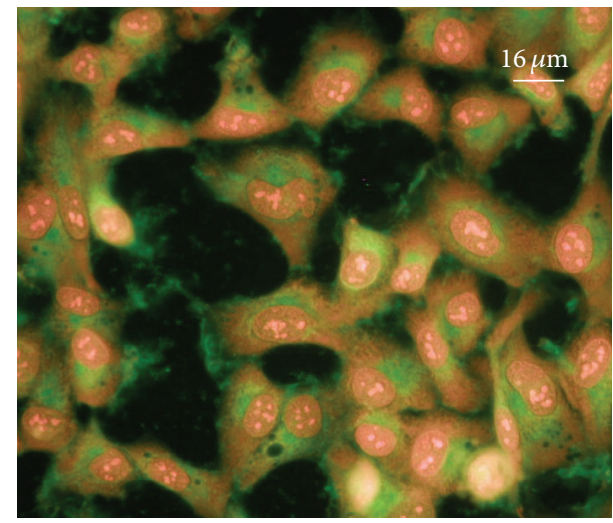

(d)

FIGURE 3: Fluorescence microscopy images relative to HT1080 cells grown for $48 \mathrm{~h}$ on the flat regions of the silicon device. Cells are fixed and double stained with FITC and PI. Blue excitation for simultaneous emission of both fluorescent probes. Images (a) and (b) are at low magnification (20x); (c) and (d) are at higher magnification (40x). Images show the spindle cell morphology typical of the mesenchymal phenotype. 


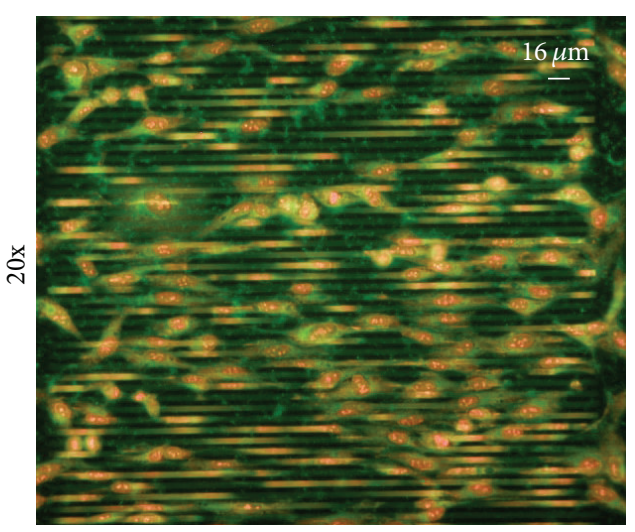

(a)

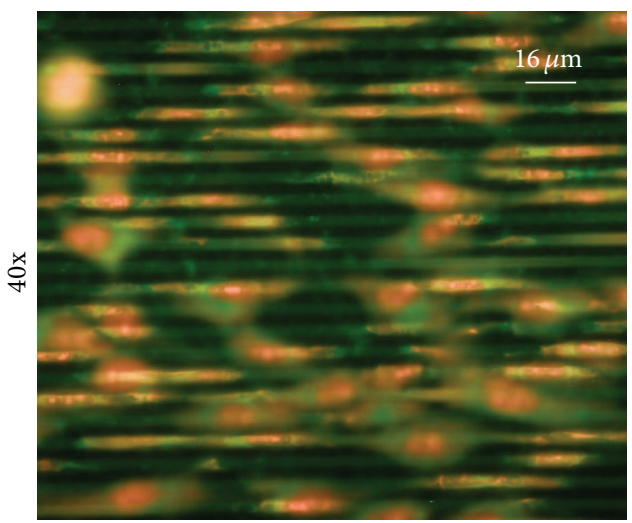

(c)

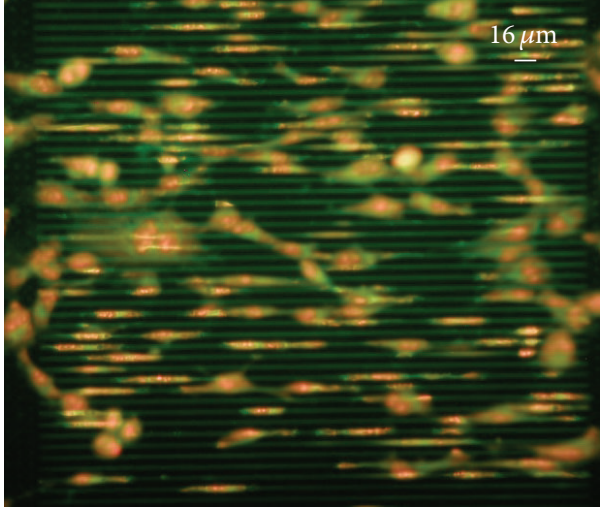

(b)

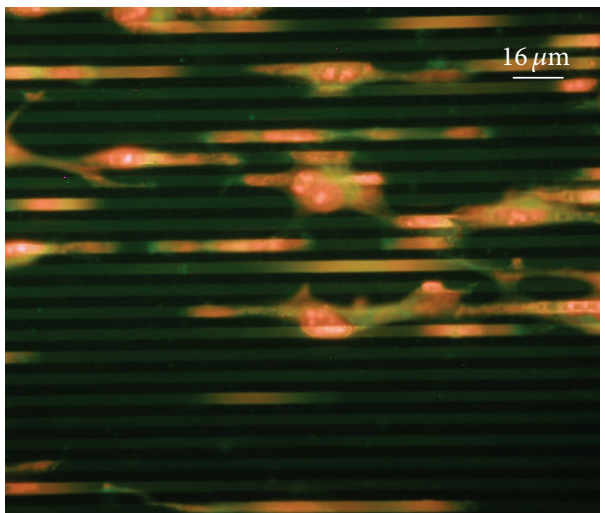

(d)

FIGURE 4: Fluorescence microscopy images relative to HT1080 cells grown for $48 \mathrm{~h}$ on the 3D-SMS. Silicon walls are horizontally oriented. The majority of the cells are squeezed inside the gaps and therefore their nuclei appear as red segments. Very few cells with normal rounded nuclei are located on top of the walls.

the SEM cross section view of a single cell cultured directly into a 3D-SMS.

2.3. Cell Culture. Human fibrosarcoma HT1080 cells were grown as monolayer in D-MEM supplemented with $10 \%$ Fetal Calf Serum (FCS), $4 \mathrm{mM}$ glutamine, $2 \mathrm{mM}$ Na pyruvate, $100 \mathrm{U} / \mathrm{mL}$ penicillin, and $0.1 \mathrm{mg} / \mathrm{mL}$ streptomycin. Cells were seeded directly on the silicon dice placed in 12 -well plates $(5$ $\times 10^{4}$ cells in $1 \mathrm{~mL}$ of medium per well). Plates were incubated at $37^{\circ} \mathrm{C}$ in a humidified atmosphere containing $5 \% \mathrm{CO}_{2}$. Increasing incubation times (until to $72 \mathrm{~h}$ ) were tested.

Reagents were purchased from Sigma Aldrich, Biowest, and Life Technologies (Milano, Italy).

2.4. Apoptosis Induction. To induce apoptosis, cells were treated with $200 \mu \mathrm{g} / \mathrm{mL}$ Bleomycin for $24 \mathrm{~h}$ and $48 \mathrm{~h}$ and further incubated in drug-free medium for additional $24 \mathrm{~h}$. The experiments were repeated at least three times in triplicate. Bleomycin was purchased from Sigma Aldrich (Milan, Italy).

2.5. Sample Fixation and Staining. After treatment, silicon was washed in Phosphate Buffered Saline (PBS) and then fixed in cold $70 \%$ ethanol for $1 \mathrm{~h}$. After fixation, the devices were washed again in PBS and incubated with RNase solution $(1 \mathrm{kUnit} / \mathrm{mL})$ for $30 \mathrm{~min}$ at $37^{\circ} \mathrm{C}$, another PBS wash was done, and then the samples were stained for $30 \mathrm{~min}$ with $1 \mu \mathrm{g} / \mathrm{mL}$ Propidium Iodide (PI) and $1 \mu \mathrm{g} / \mathrm{mL}$ Fluorescein Isothiocyanate (FITC) (both diluted in PBS). Reagents were purchased from Sigma Aldrich, Biowest, and Life Technologies (Milano, Italy).

2.6. Viability Staining. Dedicated experiments were devoted to monitor cell viability over and inside the 3D-SMS. The couple of probes PI and Hoechst 33342 (HO) were employed thanks to their peculiar characteristics: PI does not cross the intact cell membrane (typical of live cell) while $\mathrm{HO}$ is permeable to any kind of membrane. Thus, HO labels both viable and dead cells, while PI labels only the dead cell fraction [10].

2.7. Fluorescence Microscopy Analyses. At the end of the staining procedures samples were analyzed with an epifluorescent microscope BX51 (Olympus) equipped with a mercury arc 


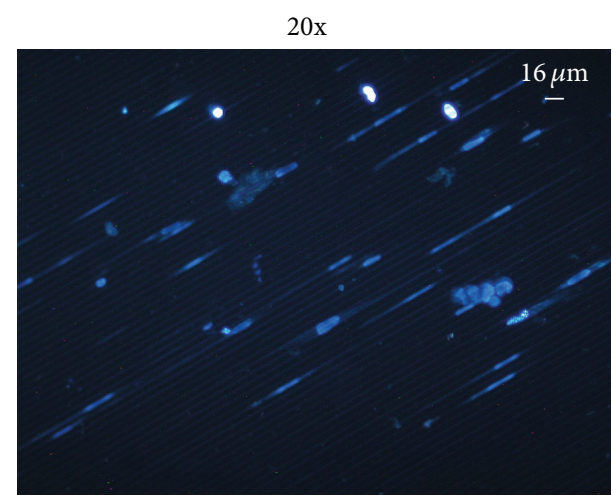

(a)

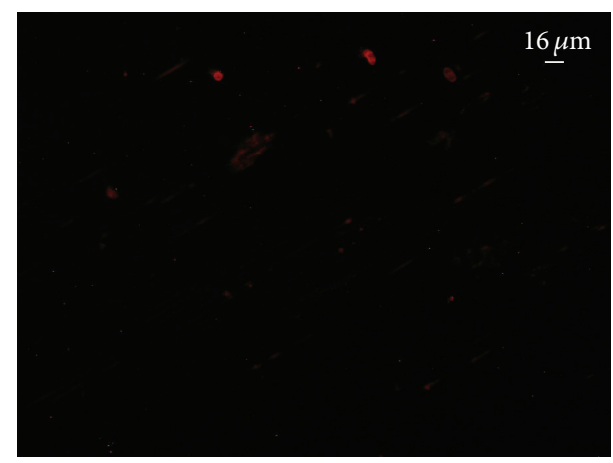

(c)

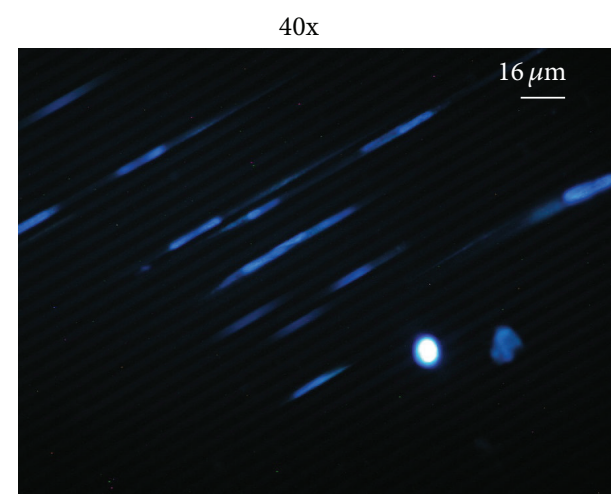

(b)



(d)

FIGURE 5: Viability test. Fluorescence microscopy images relative to HT1080 cells grown for $48 \mathrm{~h}$ on 3D-SMS (in the picture, silicon walls are oriented at $45^{\circ}$ ). Cells are supravitally stained with HO and PI (see Section 2.7) and immediately observed in UV excitation as in (a) and (b). Images show the cell nuclei emitting white-blue fluorescence. The same microscope fields are illustrated in (c) and (d) with green excitation (best for PI) to identify just a few red circular spots corresponding to the nuclei of damaged (dead) cells on top of the walls. Very likely, cells inside the gaps of 3D-SMS were not stained by PI because they were alive. (a, c) 20x; (b, d) 40x.

lamp (Osram HBO 100/2). More details are reported in [7, $8,10]$ :

(a) For fixed samples to observe both green (FITC) and red (PI) emissions, the blue excitation band was selected by an Interference Band Pass Filter (BP $450-480 \mathrm{~nm}$ ) and reflected via a Dichroic Mirror (DM 500) whereas the emitted fluorescent light was selected by means of a Barrier Long Pass Filter $515 \mathrm{~nm}$; to observe single red emission, the green excitation band was selected by an Interference Band Pass Filter (BP 530-560 nm) and reflected by a Dichroic Mirror (DM 590) whereas a Barrier Long Pass Filter (LP) $620 \mathrm{~nm}$ was used for the selection of the red emission.

(b) For viability experiments samples were observed directly immersed in the staining solution with the excitation settings, respectively, with (1) UV by means of a Band Pass Filter (BP $366 \mathrm{~nm}$ ), Dichroic Mirror (DM 400), and LP $420 \mathrm{~nm}$ as Long Pass Filter suitable for the observation of both PI and $\mathrm{HO}$ (best for $\mathrm{HO}$ ) and with (2) GREEN by means of Interference Band Pass Filter (BP 530-560 nm) and reflected by a Dichroic Mirror (DM 590) and a Barrier Long Pass Filter LP $620 \mathrm{~nm}$ used for best emission of PI.
Sample images were taken at various magnifications (Olympus U Plan Fl objectives 20x with NA of 0.50 and $40 \mathrm{x}$ with NA of 0.75 ) with the Olympus Camedia C-4040 digital camera.

\subsection{Instrumental Configuration for Label-Free Optical Detec-} tion of Cells. Label-free optical detection of cells grown in the narrow gaps of the SMS was performed by means of infrared optical low coherence reflectometry, shown as a block diagram in Figure 2. An all-fiber Michelson interferometer, reported in detail in [21-23], was implemented using two bidirectional couplers with $50: 50$ splitting ratio and flat spectral response in the wavelength range from $1.2 \mu \mathrm{m}$ to $1.7 \mu \mathrm{m}$, corresponding to the emission band of the Tungsten lamp, used as a readout source. Lamp radiation was launched partly toward the silicon device under test and partly toward a reference translating-mirror and the reflected radiation was coupled back toward the balanced receiver. The analog output signal corresponding to the interferometric fringes is the result of the interference between the fields reflected from the SMS interfaces and the reference mirror. Interferometric fringes were then recorded through an analog to digital (A/D) conversion board with a personal computer. All the experiments were carried out at a room temperature. 


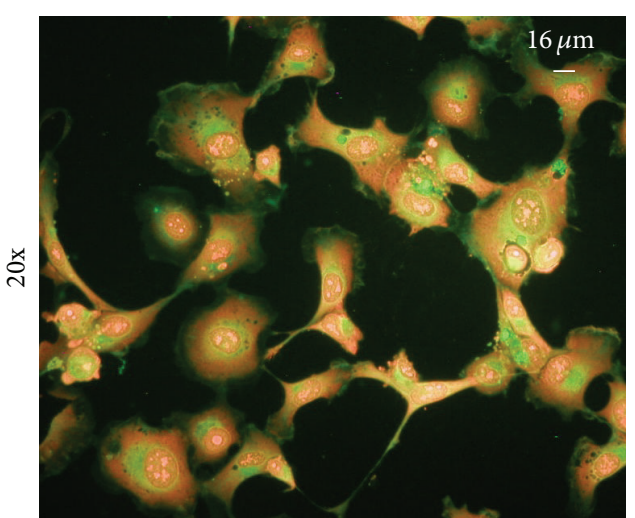

(a)

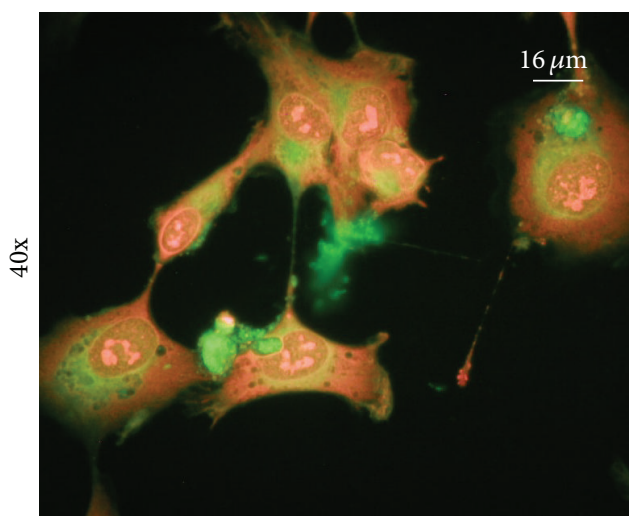

(c)

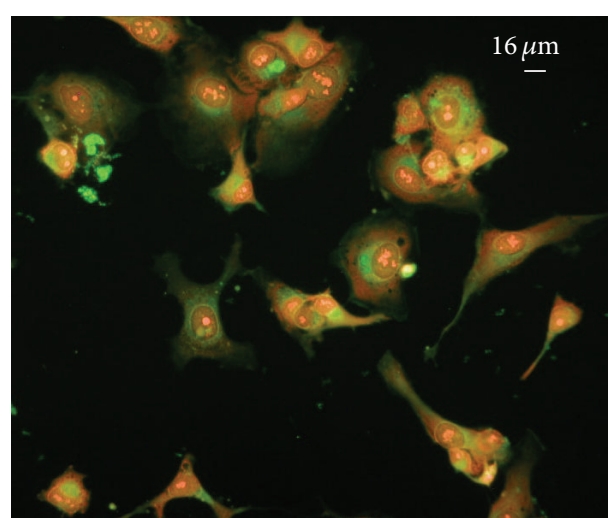

(b)

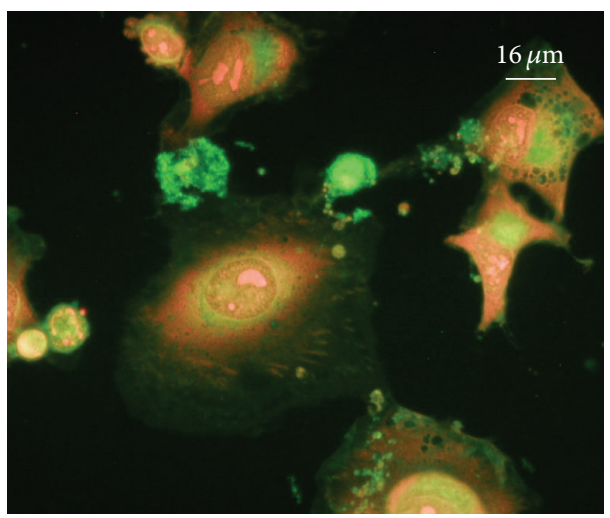

(d)

FIGURE 6: Fluorescence microscopy images relative to HT1080 cells grown for $48 \mathrm{~h}$ on the flat regions of the silicon device. Cells are here treated with Bleomycin (see Section 2.4). Images show a dramatic modification of the cell density as well as consistent changes in morphology. Figures are consistent with cells in various stages of apoptosis. (a, b) 20x; (c, d) 40x.

\section{Results and Discussion}

\subsection{Cell Culture and Apoptosis Induction}

3.1.1. Control Experiments. HT1080 cells were cultured on silicon dice in three independent experiments for $24 \mathrm{~h}$ and $48 \mathrm{~h}$ incubation times. After incubation the dice were removed from the multiwall plate, washed, fixed, stained, and finally mounted in PBS by means of a standard coverslip. Samples were then observed by fluorescence microscopy (at 20x and 40x magnification), as explained in Section 2.7, in order to evaluate the cell distribution and morphology in the various areas of the silicon die. Figure 3 reports examples of distributions of normal HT1080 cells on flat silicon regions: cells show their peculiar mesenchymal morphology. Cell proliferation rate on flat regions and morphological features and shapes were thoroughly comparable to those detected in standard growing conditions on plastic as well as on glass supports, thus under canonical 2D conditions. Fluorescence images in Figure 4 refer to cells grown on the 3D-SMS: the stretched nuclear shape (red segments) demonstrates the ability of these cells to enter the narrow gaps between silicon walls. Their mesenchymal phenotype ensures great plasticity of the cell bodies and enhanced capability to squeeze inside the narrow gaps for exploiting the vertical surface of the silicon walls. Very few cells remain adherent on top of the walls, showing a standard round nuclear shape. By comparing the cell density on the flat silicon region and on the 3D-SMS, we did not observe any significant difference (except for the stretched shape assumed by cells squeezed inside the gaps) and the proliferation rate is very similar on both regions.

3.1.2. Cell Viability. In our previous works [7, 10], we have already demonstrated that mesenchymal cells are able to enter the narrow gaps of the 3D-SMS thanks to an active process that is peculiar of this cell phenotype, committed to in vivo extreme biomechanical transitions (e.g., intravasation and extravasation). The supravital staining with $\mathrm{HO}$ allowed nuclei labeling of the whole cell population grown in the $3 \mathrm{D}$ SMS, inside the gaps, and on top of the silicon walls. On the contrary, the uptake of PI probe occurred only in damaged (dead) cells. In Figure 5 we report fluorescence images taken at 20x (a, c) and 40x (b, d) magnification. All nuclei emit blue fluorescence under UV excitation $(a, b)$ but are characterized by an elongated shape if inside the gaps or by a circular shape if on top of the silicon walls of the 3D-SMS. Green excitation of the same microscope fields $(c, d)$ revealed just 


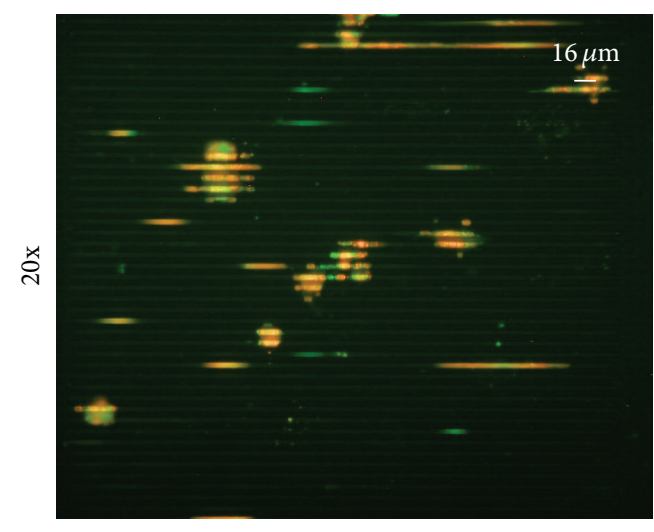

(a)

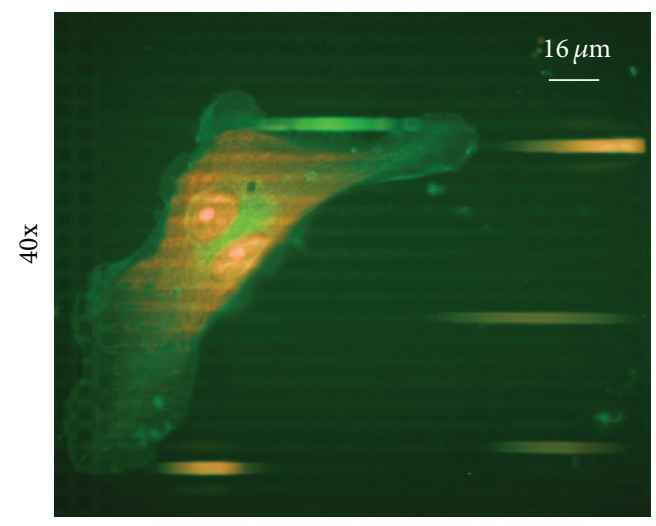

(c)



(b)

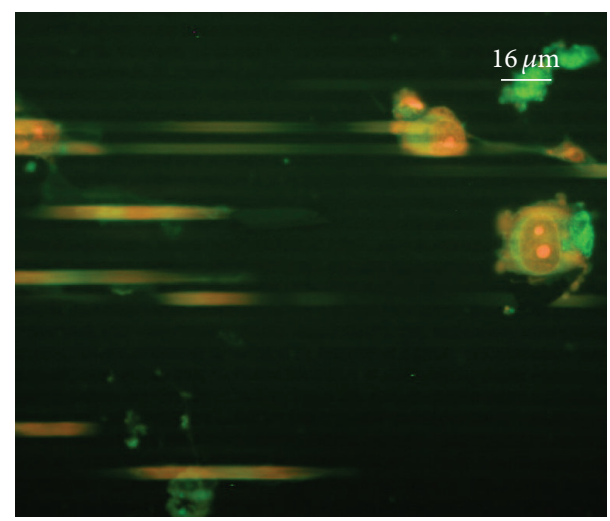

(d)

FIGURE 7: Fluorescence microscopy images relative to HT1080 cells grown for $48 \mathrm{~h}$ on the 3D-SMS (walls horizontally oriented). Cells are here treated with Bleomycin. Images show a dramatic change in both density and morphology. The drug effect reached the deepest regions of the gaps of the SMS and induced cell detachment. A few cells, which survived on the top of the walls, show typical apoptotic characteristics. (a, b) 20x; (c, d) 40x.

a few red spots corresponding to the nuclei of dead cells mainly belonging to cells located on top of the walls and on the surface of the 3D-SMS. We can conclude that the cell population grown inside the gaps of the microstructure is characterized by a high rate of viability.

\subsubsection{Apoptosis Induction Experiments. Among the tested} incubation times, we show here the results of the $48 \mathrm{~h}$ treatment with Bleomycin followed by a further $24 \mathrm{~h}$ culture in fresh medium, as this condition provided the most significant and reproducible data. The drug action is evident in terms of both important reduction of the cell density and dramatic change in cell morphology. These phenomena occurred on flat regions (Figure 6) as well as in the 3D-SMS (Figure 7). It is evident that the largest fraction of the damaged population lost the adhesion contact with the support and floated in the medium (acute phase). The surviving cell fraction, and very likely the new generated cells, proliferated in abnormal manner (late phase) as demonstrated by altered morphology. As far as the flat regions are concerned, in Figure 6 both images at 20x (a, b) and 40x (c, d) magnifications demonstrate that the drug removed most of the cells (as compared to the control) and that very few surviving adherent cells are characterized by a quite atypical shape and morphology as normally observed when cells are grown and treated in standard conditions (plastic Petri dishes/flasks or glass coverslips). Figure 7, relative to the effects on the population grown in the $3 \mathrm{D}$-SMS, shows that very few cells were able to survive inside the structure, demonstrating the drug effect even in the deep silicon microspaces. Comparing cell density and morphology, it is possible to conclude that the behavior of drug induced cell damage was very similar, indicating that Bleomycin exerts its proapoptotic effect both on the flat surface (2D) and inside the narrow gaps (3D) colonized by the cells, forcing them to detach and float in the medium.

\subsection{Label-Free Detection of a Cell in the Gap by IR OLCR.} With our setup, schematically reported in Figure 2, we successfully performed an in-plane characterization by means of horizontal and vertical scans of empty, as-fabricated silicon microstructures by measuring the optical path among several silicon/air interfaces [22]. The interferogram containing information on the optical distance between interfaces was obtained by shining the readout radiation along the $z$ axis, thus perpendicularly to the silicon walls. A typical interferometric signal (filtered and normalized) collected on 


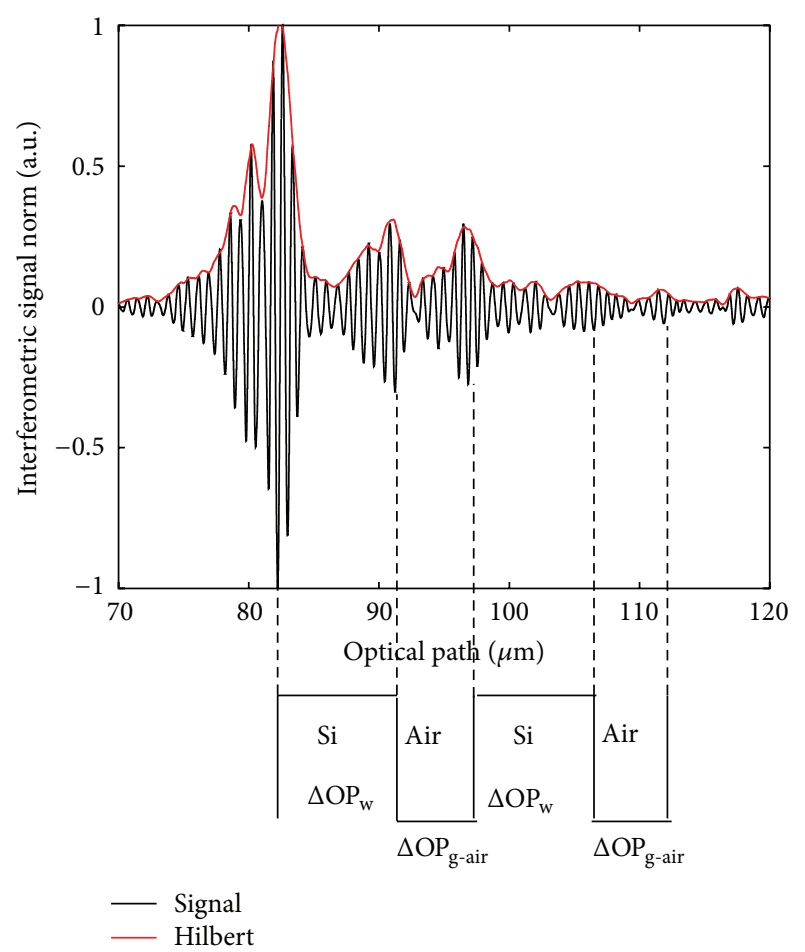

FIGURE 8: Interferometric signal, digitally filtered and normalized with respect to the peak value, collected on an as-fabricated $8 \mu \mathrm{m}$ 3D-SMS (empty gaps before cell culture). We performed also a Hilbert transform to obtain the envelope of the signal. $\Delta \mathrm{OP}_{\mathrm{g} \text {-air }}$ : optical path-length of the empty gap (only air is inside the gaps) that is obtained as the distance between the $2 \mathrm{nd}$ and $3 \mathrm{rd}$ peak (as well as between the 4 th and 5 th peak) of the envelope of the interferometric signal. $\Delta \mathrm{OP}_{\mathrm{w}}$ : optical path-length of the silicon wall that is obtained as the distance in optical path-length between the 1st and 2nd peak (as well as between the 3rd and 4th peak) of the envelope of the interferometric signal.

$8 \mu \mathrm{m}$ 3D-SMS (before cell culture) is reported as a function of the optical path inside the device in Figure 8. We also applied the Hilbert transform to extract the signal envelope. Several groups of fringes with decreasing amplitude are easily recognized: the peak of every group corresponds to the position of interfaces (air/silicon in Figure 8) crossed by the readout radiation. As it is well known, the optical path-length distance $\triangle \mathrm{OP}$ between two consecutive interfaces is given by the following relationship:

$$
\Delta \mathrm{OP}=d \cdot n,
$$

where $d$ is the geometric distance between the considered interfaces and $n$ is the group refractive index of the material or fluid present between the same interfaces. For the $8 \mu \mathrm{m} 3 \mathrm{D}$ SMS, the typical value of the optical path-length relative to a single wall is $\Delta \mathrm{OP}_{\mathrm{w}} \approx 9.4 \mu \mathrm{m}$ (i.e., the distance between the peak of the fringe groups relative to the first and the second interface) whereas for an empty gap $\Delta \mathrm{OP}_{\text {g-air }} \approx 5.4 \mu \mathrm{m}$ (i.e., the distance between the peak of the fringe groups relative to the second and the third interface). In the presence of a cell in the gap, adherent to the vertical wall, the measured optical path-length is expected to be greater than $\Delta \mathrm{OP}_{\text {g-air }}$.

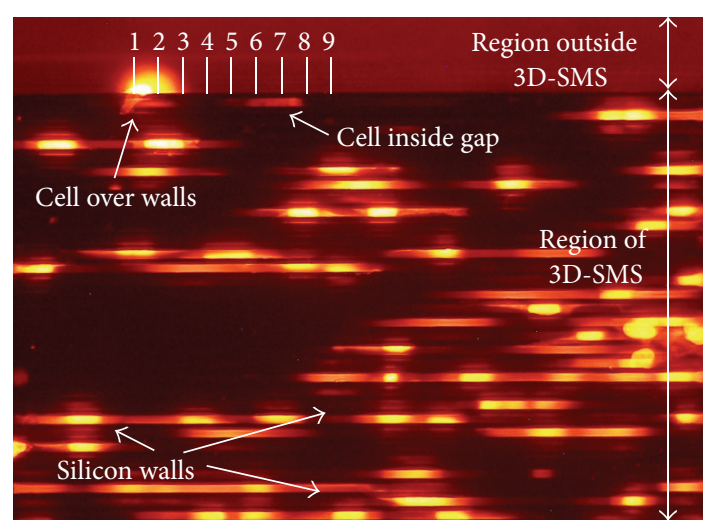

FIGURE 9: Top view of the 3D-SMS populated by cells taken with the fluorescence microscope with green excitation for PI. The bright horizontal lines are the gaps (with cells inside) separating two silicon walls. The numbers (1-9) indicate the positions where we performed low coherence interferometric measurements to detect the cell inside the first gap (corresponding to positions 6-7); they are drawn outside the 3D-SMS region so that they do not mask any part of the 3D-SMS. The "upper section" of the fluorescence image is the region outside the $3 \mathrm{D}$-SMS and does not contain significant details. The readout beam for interferometric testing arrives on the silicon device from that side.

In order to identify the effect induced by the presence of cells in the gaps, various samples of 3D-SMS were tested by means of IR OLCR, before and after cell culturing, by sequentially changing the measurement position on the target. Preliminary testing of the devices was carried out before any biological treatment, to verify the uniformity of the optical properties of the as-fabricated samples. On dice with cells, reflectometric measurements were performed by scanning the regions where fluorescence monitoring had revealed the presence of cells, deeply grown in the first gap toward the readout beam [9]. As an example, in Figure 9, we indicate the positions relative to a specific silicon micromachined die where we directed the readout beam (perpendicularly to the silicon walls) for collecting the interferometric signals. For an easier comparison of the signals relative to the various positions, we applied the Hilbert transform to extract the envelope, as shown in Figure 10 [21]. The optical path-length of the gap detected in position 7 , where a cell was identified inside the gap, is greater than that collected in position 4 relative to an empty gap zone, as better highlighted in the inset. Finally, in Figure 11, we report the optical path-length of the gap $\Delta \mathrm{OP}_{\mathrm{g}}$ and of the silicon wall $\Delta \mathrm{OP}_{\mathrm{w}}$ detected in the various positions as shown in Figure 9. As expected, the wall optical path-length $\Delta \mathrm{OP}_{\mathrm{w}}$ remains constant whereas the gap path-length $\Delta \mathrm{OP}_{\mathrm{g}}$ increases at positions 6 and 7 , thus correctly identifying the cell presence.

\section{Conclusions}

In this work, we have tested the apoptotic effects of the chemotherapeutic drug Bleomycin on HT1080 human fibrosarcoma cells grown on three-dimensional silicon microstructures with high aspect ratio. The 3D-SMS represents 




Figure 10: Envelopes of the interferometric signal obtained using Hilbert transform. The signal obtained in position 4 is typical of an empty gap situation, where only air is inside the gap, without cell inside the gap and without cell on top of walls. The signal obtained in position 7 is relative to a situation where a cell is found inside the gap, attached to the vertical silicon wall, similarly to what is reported in the SEM photos in Figure 1(b). Inset: zoom to highlight the shift of the third peak only due to the presence of a cell in the gap in that horizontal position. In presence of a cell in the gap, adherent to the vertical wall, the measured optical path-length of the gap is greater than that obtained for an empty gap.

a microenvironment for testing the effectiveness of drugs that is more challenging than standard planar surfaces, ensuring the same proliferation rate and cell viability as in standard culture conditions. The presence of narrow, deep gaps where cells have squeezed their bodies better mimics the in vivo scenery characterized by exiguous interstitial spaces. Considering that, as a major consequence of apoptosis, adherent cells tend to detach from the support where they are cultured, we have demonstrated an innovative method for detecting the presence of cells grown adherent to the vertical silicon walls. This approach will thus be suitable for monitoring the cell detachment typical of apoptosis induced by drug treatments with the same efficacy as the $2 \mathrm{D}$ condition, making possible a rapid screening of natural or synthetic drugs on cancer cells based on their potential as proapoptotic agents, thus able to kill cancer cells.

More detailed quantitative investigations will be carried out aimed at clarifying the role of 3D microenvironment in the evaluation of cell sensitivity to anticancer (proapoptotic) drugs. Our preliminary findings (obtained by the visual microscope comparison, thus operator-dependent) seem to refer a similar behavior at least for the concentration and

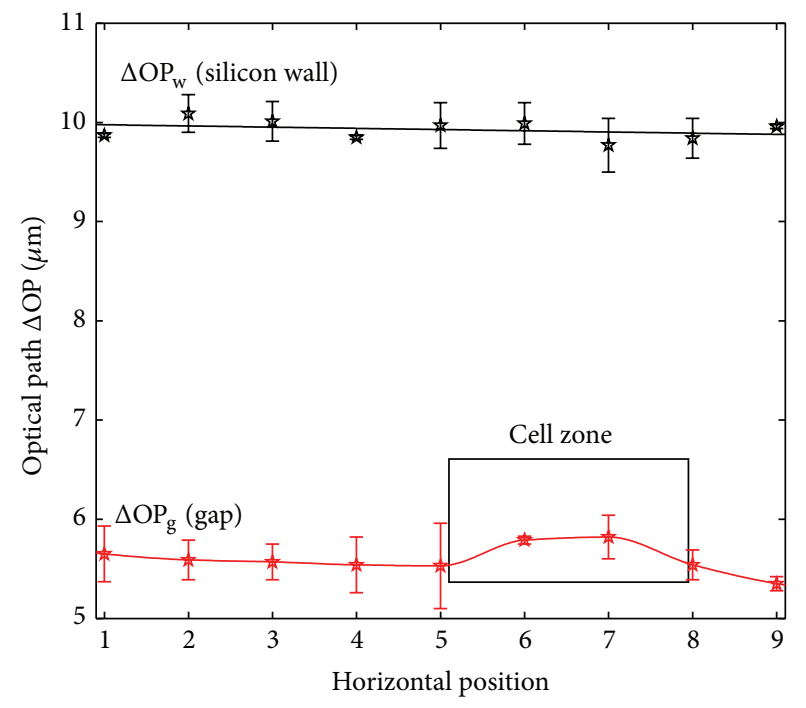

FIGURE 11: Optical path-length of the gap $\left(\Delta \mathrm{OP}_{\mathrm{g}}\right)$, that is, the distance in optical path-length between the second and third peak of the envelope of interferometric signal and of the silicon wall $\left(\Delta \mathrm{OP}_{\mathrm{w}}\right)$ measured with IR-OLCR in all the positions of the horizontal scan performed along the 3D-SMS, as shown also in Figure 9.

times generally applied in standard (2D) test conditions. It may be speculated that automated (operator independent) quantitative analyses made by means of a lab-on-a-chip system will allow to consider a wider range of concentrations and longer times for drug action. Very likely the cell in the $3 \mathrm{D}$ environment is exposed to the drug action quite differently as compared to $2 \mathrm{D}$ and perhaps will be sensitive to the damage stimuli in less time and/or at low concentration.

Finally, our innovative approach alternative to fluorescence measurements (which imply cellular toxicity) could be applied to other biomedical conditions characterized by specific cell activities and features, including the identification of CTCs (Circulating Tumor Cells) in blood, based on their peculiar biomechanical properties. In a nutshell, the 3DSMS we developed through a multidisciplinary cooperation represents a lab-on-a-chip with many possible applications in molecular oncology, combining basic research to the drug discovery field.

\section{Conflict of Interests}

The authors declare that there is no conflict of interests regarding the publication of this paper.

\section{Acknowledgment}

This work was partially supported by CARIPLO Foundation Grant no. 2011-0308. Francesca Aredia was a Ph.D. student in Genetics, Molecular and Cellular Biology, University of Pavia, Italy. 


\section{References}

[1] Y. Torisawa, H. Shiku, T. Yasukawa, M. Nishizawa, and T. Matsue, "Three-dimensional micro-culture system with a siliconbased cell array device for multi-channel drug sensitivity test," Sensors and Actuators B: Chemical, vol. 108, no. 1-2, pp. 654-659, 2005.

[2] J. H. Yeon and J. K. Park, "Microfluidic cell culture systems for cellular analysis," BioChip Journal, vol. 1, no. 1, pp. 17-27, 2007.

[3] K. F. Chambers, J. F. Pearson, N. Aziz, P. O’Toole, D. Garrod, and S. H. Lang, "Stroma regulates increased epithelial lateral cell adhesion in 3D culture: a role for actin/cadherin dynamics," PLoS ONE, vol. 6, no. 4, Article ID e18796, 2011.

[4] F. Pampaloni and E. H. K. Stelzer, "Three-dimensional cell cultures in toxicology," Biotechnology and Genetic Engineering Reviews, vol. 26, pp. 117-138, 2009.

[5] G. Barillaro, S. Merlo, S. Surdo, L. M. Strambini, and F. Carpignano, "Integrated optofluidic microsystem based on vertical high-order one-dimensional silicon photonic crystals," Microfluidics and Nanofluidics, vol. 12, no. 1-4, pp. 545-552, 2012.

[6] M. Bassu, S. Surdo, L. M. Strambini, and G. Barillaro, "Electrochemical micromachining as an enabling technology for advanced silicon microstructuring," Advanced Functional Materials, vol. 22, no. 6, pp. 1222-1228, 2012.

[7] F. Carpignano, G. Silva, S. Surdo et al., "A new cell-selective three-dimensional microincubator based on silicon photonic crystals," PLoS ONE, vol. 7, no. 11, Article ID e48556, 2012.

[8] S. Merlo, G. Barillaro, F. Carpignano et al., "Investigation of cell culturing on high-aspect-ratio, three-dimensional silicon microstructures," IEEE Journal on Selected Topics in Quantum Electronics, vol. 18, no. 3, pp. 1215-1222, 2012.

[9] S. Merlo, F. Carpignano, G. Silva et al., "Label-free optical detection of cells grown in 3D silicon microstructures," Lab on a Chip, vol. 13, no. 16, pp. 3284-3292, 2013.

[10] G. Mazzini, F. Carpignano, S. Surdo et al., “3D silicon microstructures: a new tool for evaluating biological aggressiveness of tumor cells," IEEE Transactions on NanoBioscience, vol. 14, no. 7, pp. 797-805, 2015.

[11] M. Jäättelä, "Escaping cell death: survival proteins in cancer," Experimental Cell Research, vol. 248, no. 1, pp. 30-43, 1999.

[12] V. Pavet, M. M. Portal, J. C. Moulin, R. Herbrecht, and H. Gronemeyer, "Towards novel paradigms for cancer therapy," Oncogene, vol. 30, no. 1, pp. 1-20, 2011.

[13] V. Giansanti, M. Tillhon, G. Mazzini, E. Prosperi, P. Lombardi, and A. I. Scovassi, "Killing of tumor cells: a drama in two acts," Biochemical Pharmacology, vol. 82, no. 10, pp. 1304-1310, 2011.

[14] G. Mazzini, C. Ferrari, and E. Erba, "Dual excitation multifluorescence flow cytometry for detailed analyses of viability and apoptotic cell transition," European Journal of Histochemistry, vol. 47, no. 4, pp. 289-298, 2003.

[15] S. Elmore, "Apoptosis: a review of programmed cell death," Toxicologic Pathology, vol. 35, no. 4, pp. 495-516, 2007.

[16] G. Pattabiraman, E. A. Lidstone, K. Palasiewicz, B. T. Cunningham, and D. S. Ucker, "Recognition of apoptotic cells by viable cells is specific, ubiquitous, and species independent: analysis using photonic crystal biosensors," Molecular Biology of the Cell, vol. 25, no. 11, pp. 1704-1714, 2014.

[17] C. Ma, M. Song, Y. Zhang, M. Yan, M. Zhang, and H. Bi, "Nickel nanowires induce cell cycle arrest and apoptosis by generation of reactive oxygen species in HeLa cells," Toxicology Reports, vol. 1, pp. 114-121, 2014.
[18] M. Donzelli, R. Bernardi, C. Negri et al., "Apoptosis-prone phenotype of human colon carcinoma cells with a high level amplification of the c-myc gene," Oncogene, vol. 18, no. 2, pp. 439-448, 1999.

[19] C. Gorrini, M. Donzelli, A. Torriglia et al., "Effect of apoptogenic stimuli on colon carcinoma cell lines with a different c-myc expression level," International Journal of Molecular Medicine, vol. 11, no. 6, pp. 737-742, 2003.

[20] C. R. Ross, G. Ricevuti, and A. I. Scovassi, "The antimicrobial peptide PR-39 has a protective effect against HeLa cell apoptosis," Chemical Biology and Drug Design, vol. 70, no. 2, pp. 154$157,2007$.

[21] F. Carpignano, G. Rigamonti, and S. Merlo, "Characterization of rectangular glass microcapillaries by low-coherence reflectometry," IEEE Photonics Technology Letters, vol. 27, no. 10, pp. 1064-1067, 2015.

[22] F. Carpignano, S. Surdo, G. Barillaro, and S. Merlo, "Silicon micromachined device testing by infrared low-coherence reflectometry," Journal of Microelectromechanical Systems, vol. 24, no. 6, pp. 1960-1964, 2015.

[23] G. Rigamonti, S. Merlo, and F. Carpignano, "Rectangular glass micro-capillaries for biophotonic applications," in Proceedings of the Fotonica AEIT Italian Conference on Photonics Technologies, IET Conference Proceedings, pp. 1-4, IEEE, Turin, Italy, May 2015.

[24] S. Merlo, G. Barillaro, F. Carpignano et al., "Fibrillogenesis of human $\beta 2$-microglobulin in three-dimensional silicon microstructures," Journal of Biophotonics, vol. 5, no. 10, pp. 785792, 2012. 

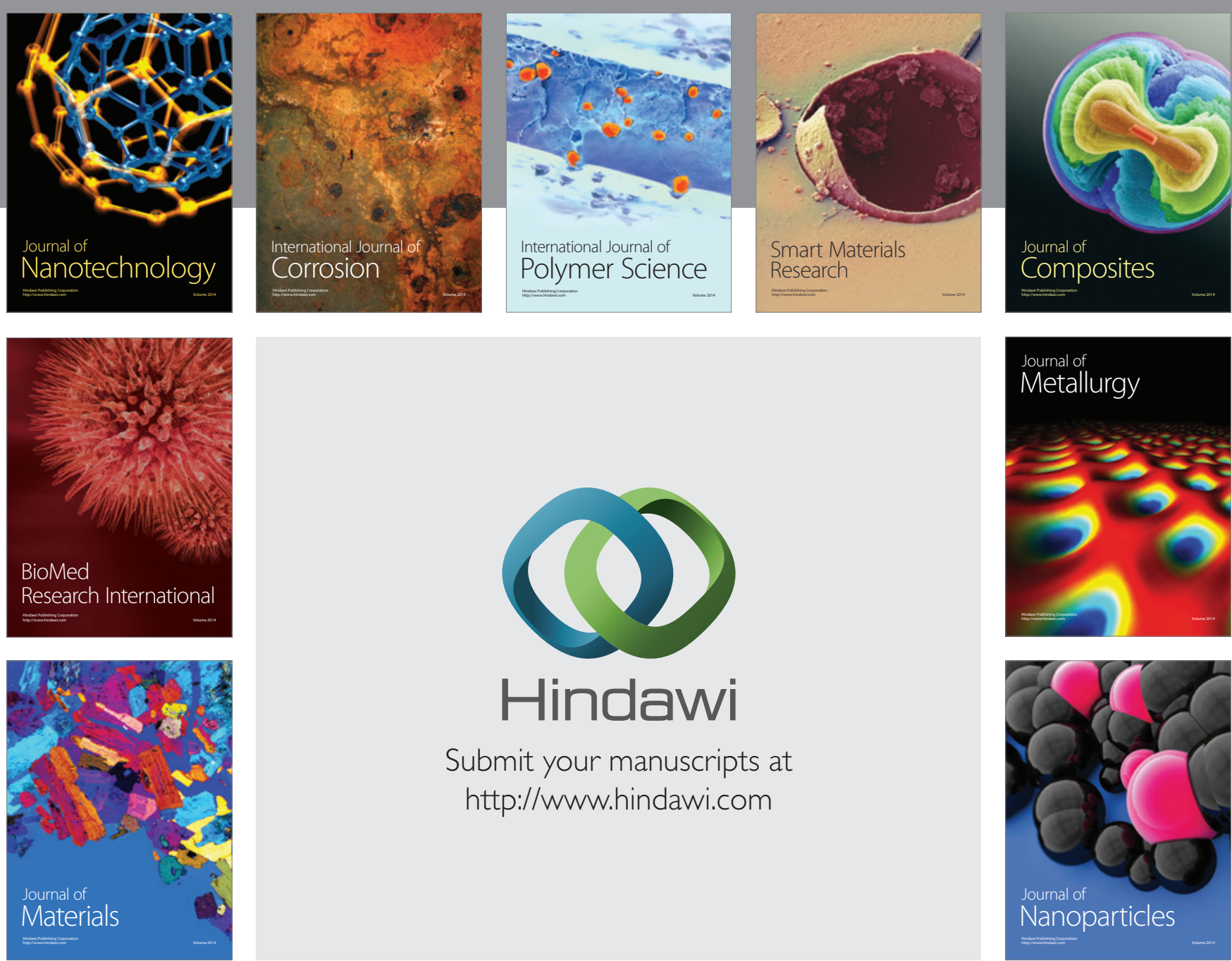

\section{Hindawi}

Submit your manuscripts at

http://www.hindawi.com

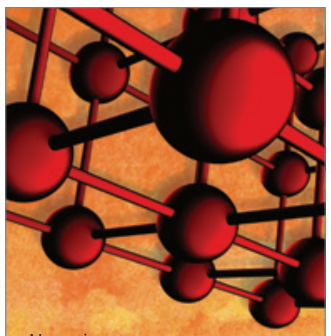

Materials Science and Engineering
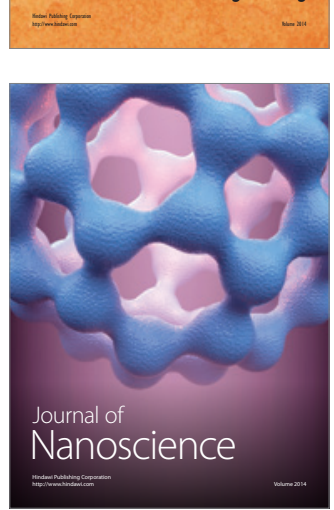
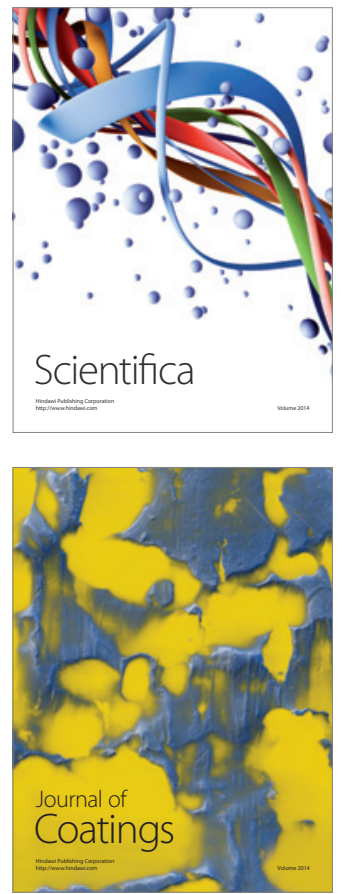
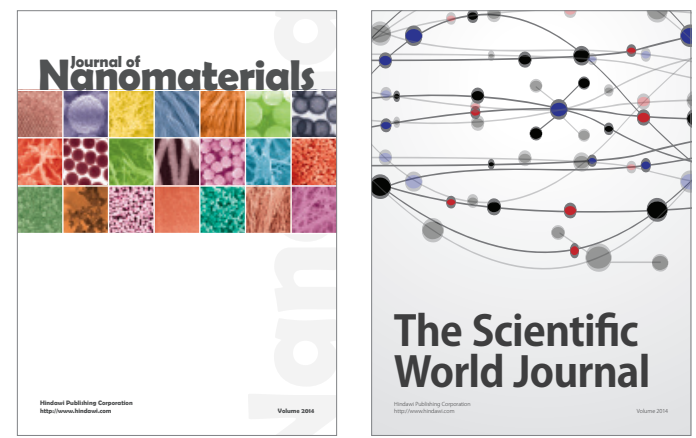

The Scientific World Journal
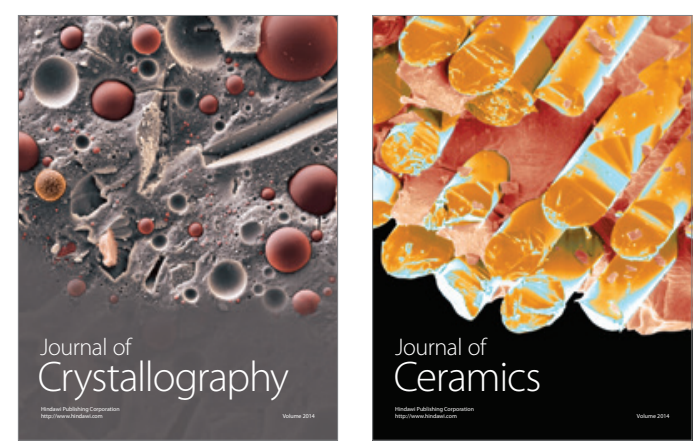
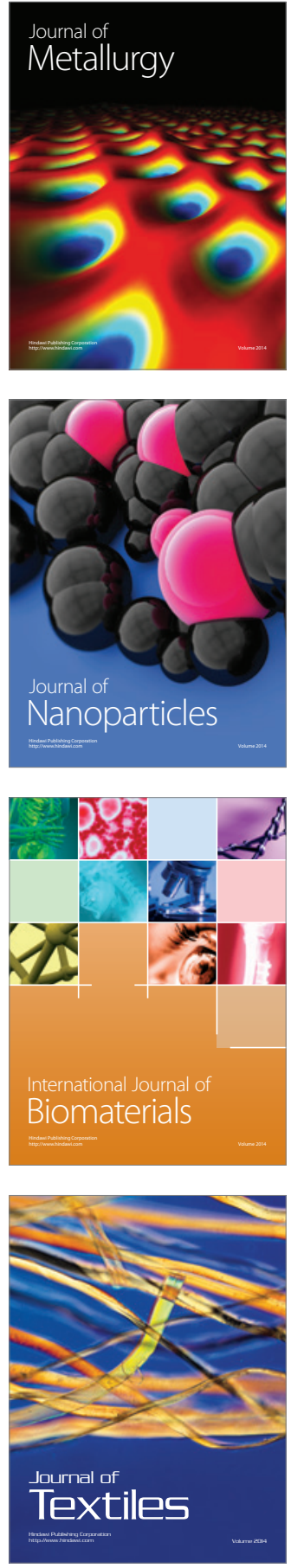